\title{
Determinants of microstructural, dimensional and bone mineral changes postpartum in Swedish women
}

\author{
Petra Brembeck*, Anna Winkvist, Claes Ohlsson, Mattias Lorentzon and Hanna Augustin \\ Department of Internal Medicine and Clinical Nutrition, Institute of Medicine, Sablgrenska Academy, University of \\ Gothenburg, Box 459, 40530 Gothenburg, Sweden
}

(Submitted 24 May 2016 - Final revision received 16 September 2016 - Accepted 11 October 2016)

\begin{abstract}
During lactation, areal (a) and volumetric (v) bone mineral density (BMD) are known to temporarily decrease. Factors that affect skeletal changes postpartum are not fully elucidated. The aim was to study determinants of the previously observed changes in aBMD at lumbar spine, and cortical vBMD, microstructure and dimensions at ultra-distal tibia postpartum. Women (25-40 years) were studied longitudinally at 2 weeks (baseline) and 4 months ( $n$ 81), 12 months ( $n$ 79) and 18 months ( $n 58)$ postpartum. At each visit, blood samples were collected, body weight and height were measured and information about lactation habits, oestrogen contraceptives and physical activity was obtained. Ca intake was measured using 4 -d food diaries at 4 months postpartum. Serum 25-hydroxyvitamin D (25OHD) was analysed by liquid chromatography-tandem MS. Skeletal changes were assessed with dual-energy X-ray absorptiometry and high-resolution peripheral quantitative computed tomography. Mean baseline BMI was 24.8 (sD $3 \cdot 1) \mathrm{kg} / \mathrm{m}^{2}$. Median (quartiles 1-3) duration of total lactation was $8 \cdot 1$ (6.8-10.4) months. Longer duration of full lactation was associated with larger decreases of lumbar spine aBMD and tibia vBMD and microstructure. Higher baseline body weight was associated with smaller decreases in tibia vBMD and microstructure. Higher Ca intake was associated with smaller decreases in tibia cortical vBMD and thickness. Higher baseline 25OHD was only associated with larger decreases in lumbar spine aBMD. In conclusion, lactation and body weight were the main determinants of skeletal changes during the first 18 months postpartum. $\mathrm{Ca}$ intake and serum concentrations of $25 \mathrm{OHD}$ appear to have different associations with cortical and trabecular bone.
\end{abstract}

Key words: Bone mineral density: Lactation: Postpartum: 25-Hydroxyvitamin D: Vitamin D: Dual-energy X-ray absorptiometry: High-resolution peripheral quantitative computed tomography

Areal bone mineral density (aBMD) is known to temporarily decrease during lactation ${ }^{(1-11)}$. About $200-400 \mathrm{mg} \mathrm{Ca}$ is required for breast milk production per day ${ }^{(3)}$. The largest decreases postpartum in aBMD (approximately 2-6\%) are found at the lumbar spine and femoral neck ${ }^{(1-4,7,10,11)}$. Previous studies have also demonstrated a relationship between longer duration of lactation and larger decreases in $\operatorname{aBMD}^{(2,3,5,7-11)}$. In addition, it has been found that the decrease in aBMD at the lumbar spine occurs early postpartum among lactating women, whereas for women with long duration of lactation the decrease has been observed in femoral neck aBMD a few months later ${ }^{(6,7,10,11)}$. This has led to the assumption that decreases in aBMD initially occur in the trabecular-rich bones. However, women with extended duration of lactation show a later decrease in aBMD in cortical-rich bones.

Most previous studies have used dual-energy X-ray absorptiometry (DXA) to study skeletal changes postpartum, and have thus reported $\operatorname{aBMD}^{(2,3,7,10,11)}$. However, there are indications that high-resolution peripheral quantitative computed tomography (HR-pQCT) may detect small changes not detectable by DXA ${ }^{(12)}$.
Furthermore, HR-pQCT measures volumetric bone mineral density (vBMD), distinguishes between cortical and trabecular bone and provides additional information about microstructural changes such as trabecular thickness and dimensional changes such as cortical thickness ${ }^{(12)}$. We have recently shown in the BUGA (Benmetabolism Under Graviditet och Amning; Bone Metabolism During Pregnancy and Lactation) study that in women lactating 4 months or longer cortical vBMD and cortical thickness at ultradistal tibia decreased significantly during the first 12 months postpartum $^{(11)}$. In women lactating for less than 4 months, no significant decreases were found in these bone variables during the first 18 months postpartum.

At the end of lactation or after weaning, most studies show a recovery of aBMD ${ }^{(10,11,13-15)}$. However, it is still not clear whether the skeleton fully recovers at all skeletal sites, nor whether it fully recovers in women with extended lactation ${ }^{(16)}$. Affinito et $a l^{(16)}$ reported incomplete recovery of aBMD at the lumbar spine at 6 months after lactation cessation. In the BUGA study, we have shown that for women lactating 9 months or longer, cortical vBMD and trabecular thickness at ultra-distal tibia were

Abbreviations: 25OHD, 25-hydroxyvitamin D; aBMD, areal bone mineral density; BUGA, Benmetabolism Under Graviditet och Amning (Bone Metabolism During Pregnancy and Lactation); PAL, physical activity level; vBMD, volumetric bone mineral density.

* Corresponding author: P. Brembeck, email Petra.Brembeck@gu.se 
still significantly lower at 18 months postpartum compared with shortly after delivery ${ }^{(11)}$. Besides lactation, determinants of changes in aBMD postpartum have rarely been studied and determinants of changes in vBMD, microstructural and dimensional bone parameters postpartum have not been studied at all. Vitamin D is known to increase intestinal Ca uptake, Ca resorption from the skeleton and $\mathrm{Ca}$ resorption from the kidneys $^{(17)}$. Investigations of associations between maternal vitamin $\mathrm{D}$ intake and aBMD postpartum are rare ${ }^{(13)}$. Krebs et $a l .{ }^{(13)}$ found no association between dietary vitamin D intake and lumbar spine or mid-radius aBMD postpartum. We are unaware of any previous study that has investigated the relationship between vitamin $\mathrm{D}$ status (as assessed by the biochemical marker 25-hydroxyvitamin D (25OHD) in serum or plasma) and changes in aBMD, vBMD or microstructural and dimensional bone parameters during lactation.

Most studies investigating the relation between $\mathrm{Ca}$ intake and changes in aBMD postpartum found no relationship between these parameters ${ }^{(2,3,14)}$. In contrast, Krebs et al. ${ }^{(13)}$ found a positive association between total dietary $\mathrm{Ca}$ intake and lumbar spine aBMD postpartum. Higher body weight has previously been associated with smaller changes in aBMD at various skeletal sites during pregnancy ${ }^{(18)}$. However, no association between body weight or change in body weight and changes in proximal femur and lumbar spine aBMD has been found postpartum $^{(2,14)}$. Serum oestradiol levels have, however, been positively associated with lumbar spine aBMD postpartum ${ }^{(13)}$. Other factors that have been reported to be inversely associated with decreases in aBMD at various skeletal sites postpartum include parity ${ }^{(6,13)}$, maternal height ${ }^{(3)}$ and maternal age ${ }^{(6,19)}$.

Thus, factors determining the skeletal changes postpartum are not fully elucidated. Moreover, there are no other studies investigating determinants of changes in vBMD, microstructural and dimensional parameters postpartum. Thus, within the BUGA study, we aim to investigate determinants of the observed significant changes in aBMD at the lumbar spine, and vBMD as well as microstructural and dimensional parameters at the ultradistal tibia in women between 2 weeks and 18 months postpartum.

\section{Methods}

\section{Subjects}

In all, eighty-one pregnant women were recruited to the BUGA study from July 2008 to July 2011 using posters in maternity health care clinics and in public places near Gothenburg, Sweden, and through advertisements on a Swedish webpage addressing pregnant women in western Sweden, as previously described $^{(20)}$. Inclusion criteria were women within the age range of 25-40 years, and declaring oneself healthy. Exclusion criteria were intake of prescribed medicine known to affect $\mathrm{Ca}$ and bone metabolism, recent bone fractures, pregnancy during the past 1.5 years before current pregnancy, miscarriage after week 12 of pregnancy during the past 1.5 years, breast-feeding during the past year before current pregnancy, current twin pregnancy and current development of gestational diabetes or preeclampsia. This study was conducted according to the guidelines laid down in the Declaration of Helsinki ${ }^{(21)}$, and all procedures involving the subjects were approved by the Regional Ethics Committee in Gothenburg and the Swedish Radiation Safety Authority. Written informed consent was obtained from all women.

\section{Study design}

All women were invited to visit the Department of Internal Medicine and Clinical Nutrition, University of Gothenburg, Sweden, within 2 weeks after delivery (baseline) and 4, 12 and 18 months after delivery. Venous blood was drawn in the morning after an overnight fast at all visits. Body weight in underwear was measured (Tanita, BWB-800MA; Rex Frederiksbergs Vaegtfabrik) and height was assessed using a standardised wall stadiometer at all visits. At all visits, women were asked about their lactation habits, physical activity level (PAL) and use of oestrogen contraceptives. The changes in vitamin D status postpartum in these women ${ }^{(20)}$ and the changes in bone variables postpartum ${ }^{(11)}$ have been reported previously, separately. In this paper, we analyse the determinants (including serum 25OHD and Ca intake) of the observed significant microstructural, dimensional and bone mineral changes postpartum in these women.

\section{Methods}

At all visits, bone status was measured at the Osteoporotic Unit at the Geriatric Medicine Clinic, Sahlgrenska University Hospital, Gothenburg, Sweden. Lumbar spine aBMD was measured by DXA (Lunar Prodigy, software version 11.400.004; GE Lunar Corp.). Cortical vBMD and cortical and trabecular thickness were measured at the ultra-distal tibia by HR-pQCT (XtremeCT, software version 5.3; Scanco Medical AG), as previously described $^{(11)}$. If the woman was lactating, detailed information about lactation habits was collected at all study visits, including number of lactation sessions per day, number and amount of formula feedings per day, date of introduction of solid foods and daily amount of solid foods given. Women were asked to record the date of lactation cessation. Duration of total lactation refers to duration of any type of lactation (full and partial). Duration of full lactation was defined as when $\geq 90 \%$ of the infants' daily energy intake came from breast milk. At 4 months, lactation was categorised as full lactation (yes $=1)$ or not $($ no $=0)$. At 12 and 18 months postpartum, lactation was defined as the following continuous variables: duration of full lactation in months (at 12 months postpartum) and duration of total lactation in months (at 18 months postpartum).

Dietary $\mathrm{Ca}$ intake at 4 months postpartum was estimated using 4-d food diaries, as described elsewhere ${ }^{(22)}$. In brief, women were asked to record all food and drink consumed as precisely as possible on 4 consecutive days with at least 1 nonworking day, starting no later than 1 week after the study visit. Both oral and written information were given on how to fill in the food diary. Women were asked to report the amounts of consumed food items using household measures, weight (grams) or using photographs of different portion sizes used in the Swedish portion guide 'Matmallen',(23). Women were also asked not to change their diet. Women were contacted if any ambiguities were noted in their food diaries. Dietary intake was 
calculated using DietistXP, version 3.1 (The National Food Agency food database version 2009-11-10; Kost och näringsdata). Details of use, frequency, amount and brand of supplements containing $\mathrm{Ca}$ were also requested. For the statistical analyses, total dietary $\mathrm{Ca}$ intake was used, including both dietary $\mathrm{Ca}$ intake and intake of supplements containing Ca.

Each woman rated her physical activity on a scale between 1 and 10 , as previously described ${ }^{(22)}$. In brief, women were informed that 1 indicated a sedentary lifestyle, 5 a few long walks each week and 10 exercise several times a week. The answer was converted to PAL, where 1 corresponded to PAL $1 \cdot 3$ and 10 to PAL $2 \cdot 2$, respectively. Each step in between represented a 0.1 increase in PAL. In a validation study, PAL assessed using this scale was correlated $(r 0.54 ; P=0.008)$ with corresponding estimates obtained using criterion methods (i.e. the doubly labelled water method in combination with indirect calorimetry) in twenty-two healthy Swedish pregnant women (Marie Löf, personal communication).

\section{Laboratory analyses}

Blood samples were protected from UVB light and centrifuged within $45 \mathrm{~min}$ of sampling at $5^{\circ} \mathrm{C}, 3800 \mathbf{g}$, for $9 \mathrm{~min}$ (Centrifuge CR3i; Jouan Quality System). Serum was then aliquoted and stored at $-70^{\circ} \mathrm{C}$ until analysis. The analyses of serum concentrations of $25 \mathrm{OHD}_{3}$ at all visits postpartum were performed in batches using liquid chromatography-MS (Mass spectrometer API 4000) by Central Laboratory in Malmö, Sweden, which is affiliated to the Vitamin D External Quality Assessment Scheme ${ }^{(24)}$. The method has a measuring range of $6-450 \mathrm{nmol} / \mathrm{l}$ for $25 \mathrm{OHD}_{3}$. This method has an inter-CV of $6 \%$ at $40 \mathrm{nmol} / \mathrm{l}$.

\section{Statistical analyses}

Percentage change in bone variables compared with baseline was calculated by the difference in log-transformed data between two time points, using $\log e$ and multiplied by 100 , as previously described ${ }^{(11)}$. This approximates the percentage change, as shown by Cole ${ }^{(25)}$.

Univariable linear regression analyses were first used to evaluate the relationships between percentage change in each bone parameter (lumbar spine aBMD, cortical vBMD, cortical thickness and trabecular thickness) at 4, 12 and 18 months postpartum, respectively, compared with baseline, and possible determinants. The possible determinants included body weight at baseline, change in body weight (time of measurement minus baseline), height, lactation, age, parity, serum 25OHD at baseline, total $\mathrm{Ca}$ intake at 4 months postpartum, PAL at 4 months postpartum, use of contraceptives containing oestrogen at 4 months postpartum and baseline bone value. All variables found to be significant in the univariable linear regression analyses were entered into a multivariable regression analysis also adjusted for lactation, body weight at baseline, change in body weight at the time of measurement minus baseline and height. At 4 months postpartum, lactation was used as a categorical variable (full lactation (yes $=1)$ or not $($ no $=0)$ ), as at that time point a majority of the women were still fully lactating. At 12 months postpartum, lactation was used as a continuous variable with duration of full lactation in months, as at that time point a considerable number of the women were still lactating to some extent. At 18 months postpartum, lactation was used as the continuous variable duration of total lactation in months.

As bone variables were not normally distributed, these variables were log-transformed before further analysis. Percentage change in bone variables was calculated according to the method of Cole ${ }^{(25)}$ as the difference in log-transformed data at 4, 12 or 18 months postpartum minus baseline, using $\log e$, and multiplied by 100 . In the regression analyses, PAL and use of hormonal contraceptives containing oestrogen at 4 months postpartum were included in the analysis, as this time point probably more accurately reflects the actual situation during the first 4 months postpartum than do the baseline values.

Statistical analyses for comparisons between women fully lactating and women not fully lactating at 4 months postpartum was performed with Students' $t$ test.

Co-linearity was evaluated between serum 25OHD and season and a positive co-linearity was found. Therefore, season was not included as a possible determinant in the model. However, interactions between serum 25OHD at baseline, and lactation category at 4 months postpartum, as well as serum 25OHD and season at baseline, were analysed as combined variables in the multivariable regression analyses for lumbar spine aBMD at 4 months postpartum. In the same way, interactions between total $\mathrm{Ca}$ intake at 4 months postpartum and duration of total lactation was analysed in the multivariable regression analyses for cortical vBMD and cortical thickness at the ultra-distal tibia at 18 months postpartum. No such significant interactions were found.

A two-tailed $P$ value $<0.05$ was considered statistically significant. All analyses were conducted using SPSS Statistics Software, version 22.0; IBM. Means and standard deviations were used, unless otherwise described.

\section{Results}

Descriptive characteristics of the participating women are shown in Table 1 . The number of women participating in the study was eighty-one at baseline and at 4 months postpartum, seventy-nine women at 12 months postpartum and fifty-eight women at 18 months postpartum. Reasons for leaving the study were new pregnancy ( $n$ 14), time constraints $(n 6)$ and development of disease $(n$ 3).

The mean age of the women was 32.9 (sD 3.4) years (range 25-40 years) and the mean height was $168.4(\mathrm{sD} 6.4) \mathrm{cm}$. All births were term births. Parity at recruitment prepartum ranged from 0 to 2 . In all, $80 \%$ of the women had studied for 3 years or more at university level. No women were smoking at baseline and only three (4\%) women were smoking at 12 months postpartum. Median duration (quartiles 1-3) of full lactation was $5 \cdot 0(3 \cdot 0-6 \cdot 1)$ months and median duration of total lactation was $8 \cdot 1(6 \cdot 8-10 \cdot 4)$ months. At 2 weeks postpartum, $90 \%$ of the women were fully lactating and $98 \%$ were lactating to some extent. At 4 months postpartum, $70 \%$ of the women were fully lactating and $88 \%$ were lactating to some extent. At 12 months postpartum, none of the women were fully lactating, but $16 \%$ of the women were lactating to some extent. At 18 months 
Table 1. Descriptive characteristics of the participating women*

(Mean values and standard deviations)

\begin{tabular}{|c|c|c|c|c|c|c|c|c|c|c|c|}
\hline \multirow{3}{*}{$\frac{\text { Descriptives }}{\text { Lactation } \ddagger}$} & \multirow{2}{*}{\multicolumn{2}{|c|}{ Baseline† }} & \multicolumn{4}{|c|}{4 months postpartum } & \multirow[b]{3}{*}{$P$} & & & & \\
\hline & & & \multicolumn{2}{|c|}{ No } & \multicolumn{2}{|c|}{ Yes } & & \multicolumn{2}{|c|}{12 months postpartum } & \multicolumn{2}{|c|}{18 months postpartum } \\
\hline & Mean & SD & Mean & SD & Mean & SD & & Mean & SD & Mean & SD \\
\hline$n$ & \multicolumn{2}{|c|}{81} & \multicolumn{2}{|c|}{24} & \multicolumn{2}{|c|}{57} & & \multicolumn{2}{|c|}{79} & \multicolumn{2}{|c|}{58} \\
\hline Age (years) & & & 31.8 & $2 \cdot 7$ & $33 \cdot 4$ & 3.6 & 0.060 & & & & \\
\hline Body weight (kg) & $70 \cdot 2$ & 3.4 & 66.9 & $9 \cdot 2$ & 66.9 & 8.3 & 0.988 & $65 \cdot 3$ & 8.9 & $65 \cdot 2$ & 8.6 \\
\hline BMI $\left(\mathrm{kg} / \mathrm{m}^{2}\right)$ & 24.8 & 3.1 & 23.9 & 3.7 & 23.5 & $2 \cdot 6$ & 0.572 & 23.1 & 3.0 & 23.0 & 2.6 \\
\hline PAL§ & 1.6 & 0.2 & $1 \cdot 8$ & 0.2 & $1 \cdot 8$ & 0.2 & 0.293 & 1.8 & 0.2 & 1.8 & 0.2 \\
\hline Serum 25OHD (nmol/l) & 68 & 24 & $72 \cdot 9$ & $26 \cdot 7$ & $75 \cdot 3$ & $20 \cdot 3$ & 0.659 & 67 & 19 & 71 & 20 \\
\hline
\end{tabular}

PAL, physical activity level; 25OHD, 25-hydroxyvitamin D.

* Statistical analyses for comparisons between women fully lactating and women not fully lactating at 4 months postpartum were performed with Students' $t$ test.

† Baseline; 2 weeks postpartum.

‡ Lactation; fully lactating at 4 months postpartum: no or yes.

$\S$ PAL (range 1.3-2.2).

postpartum, only one woman was still lactating to some extent.

At 4 months postpartum, 5\% of the women were using oestrogen contraceptives, at 12 months postpartum $7 \%$ of the women were using oestrogen contraceptives and at 18 months postpartum $9 \%$ of the women were using oestrogen contraceptives. The majority of the women were feeding the infant at the breast, not expressing breast-milk to be fed to the infant, also during long lactation.

Mean dietary Ca intake was 1110 (sD 410) mg/d at 4 months postpartum. In all, $33 \%$ of the women were taking supplements containing $\mathrm{Ca}$ at 4 months postpartum. Mean serum 25OHD was above $50 \mathrm{nmol} / 1$ at all time points postpartum. In all, $39 \%$ of the women were taking supplements containing vitamin $\mathrm{D}$ at baseline and $31 \%$ of the women at 4 months postpartum.

As previously reported, significant changes postpartum were found in aBMD at the lumbar spine and in cortical vBMD, cortical thickness and trabecular thickness at the ultra-distal tibia, in this population of women ${ }^{(11)}$. Determinants of these changes in bone variables postpartum are described below.

\section{Determinants of changes in areal bone mineral density at the lumbar spine}

During the first 4 months postpartum, lumbar spine aBMD decreased significantly $(-3.48$ (SD 0.44$) \%, P<0.001)^{(11)}$, as shown in Fig. 1. At 4 months postpartum, baseline body weight $(P=0.021)$ was positively associated with changes in lumbar spine aBMD, and serum 25OHD $(P=0.003)$ and aBMD $(P=0.012)$ were inversely associated with changes in lumbar spine aBMD (Table 2), as analysed with multivariable linear regression model.

At 12 months postpartum, lumbar spine aBMD had increased significantly compared with baseline $(+1 \cdot 21$ (SD 0.45$) \%$, $P<0.001)^{(11)}$. Durations of full lactation $(P=0.002)$ and baseline aBMD $(P<0 \cdot 001)$ were inversely associated with the changes in lumbar spine aBMD during the first 12 months postpartum (Table 2), as analysed with multivariable linear regression model.

At 18 months postpartum, lumbar spine aBMD had increased significantly with $+3.51 \quad(\mathrm{sD} \quad 0.47) \% \quad(P<0.001)$ compared

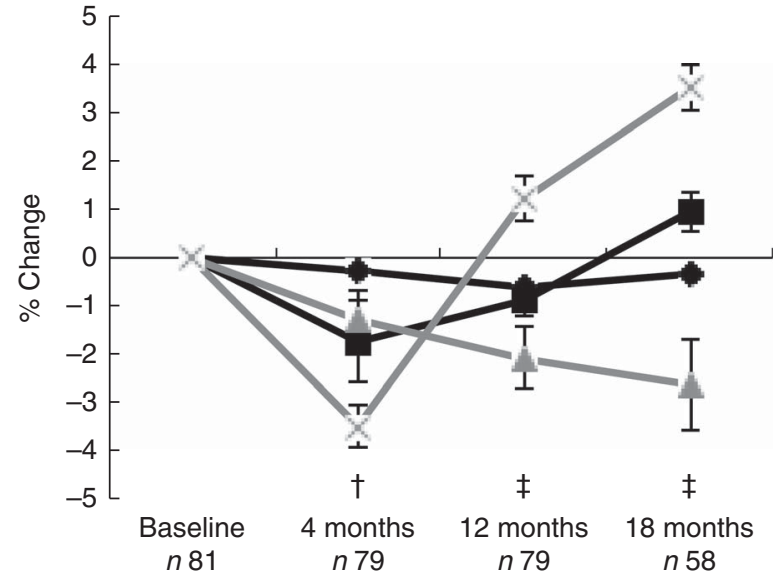

Fig. 1. Mean percentage change from baseline (standard errors) in log bone variables in women postpartum, as assessed with dual-energy X-ray absorptiometry and high-resolution peripheral quantitative computed tomography. $†$ Significant change compared with baseline for areal bone mineral density (aBMD) at the lumbar spine and for cortical volumetric bone mineral density (vBMD) and cortical thickness at the ultra-distal tibia. ¥ Significant change compared with baseline for aBMD at the lumbar spine and for cortical $\mathrm{VBMD}$, cortical thickness and trabecular thickness at the ultradistal tibia. $\longrightarrow$, Cortical VBMD; $\square-$, cortical thickness; $\longrightarrow$, trabecular thickness; $\longrightarrow$, lumbar spine aBMD.

with baseline ${ }^{(11)}$. A significant inverse relationship was still found between baseline aBMD at lumbar spine and changes in lumbar spine aBMD during the first 18 months postpartum $(P<0.001)$ (Table 2), as analysed with multivariable linear regression model.

\section{Determinants of changes in cortical volumetric bone mineral density at ultra-distal tibia}

During the first 4 months postpartum, cortical vBMD at the ultra-distal tibia decreased significantly $(-0 \cdot 28$ (SD $0 \cdot 06) \%$, $P<0.001)$ (Fig. 1) ${ }^{(11)}$. Both body weight at baseline $(P=0.006)$ and use of oestrogen contraceptives at 4 months postpartum $(P=0.046)$ were positively associated with changes in cortical vBMD during the first 4 months postpartum (Table 3 ), as analysed with multivariable linear regression model. 
Table 2. Determinants for percentage change compared with baseline† in lumbar spine areal bone mineral density (aBMD) postpartum ( $\beta$-Coefficients with their standard errors)

\begin{tabular}{|c|c|c|c|c|c|c|c|c|c|c|c|c|}
\hline \multirow[b]{3}{*}{ Determinants } & \multicolumn{6}{|c|}{ Univariable linear regression } & \multicolumn{6}{|c|}{ Multivariable linear regression } \\
\hline & \multicolumn{2}{|c|}{$4 \mathrm{mo} \mathrm{pp}$} & \multicolumn{2}{|c|}{$12 \mathrm{mo} \mathrm{pp}$} & \multicolumn{2}{|c|}{$18 \mathrm{mo} \mathrm{pp}$} & \multicolumn{2}{|c|}{$4 \mathrm{mo} \mathrm{pp}$} & \multicolumn{2}{|c|}{$12 \mathrm{mo} \mathrm{pp}$} & \multicolumn{2}{|c|}{$18 \mathrm{mo} \mathrm{pp}$} \\
\hline & $\beta$ & SEM & $\beta$ & SEM & $\beta$ & SEM & $\beta$ & SEM & $\beta$ & SEM & $\beta$ & SEM \\
\hline$n$ & \multicolumn{2}{|c|}{81} & \multicolumn{2}{|c|}{79} & \multicolumn{2}{|c|}{58} & \multicolumn{2}{|c|}{81} & \multicolumn{2}{|c|}{79} & \multicolumn{2}{|c|}{58} \\
\hline Body weight $(\mathrm{kg}) \dagger$ & +0.066 & 0.046 & +0.013 & 0.048 & +0.013 & 0.050 & $+0 \cdot 117$ & $0.50^{*}$ & +0.66 & 0.047 & +0.078 & 0.058 \\
\hline Body weight change $(\mathrm{kg}) \ddagger$ & +0.001 & 0.123 & +0.085 & 0.107 & +0.076 & 0.104 & -0.001 & 0.118 & +0.096 & 0.096 & $+0 \cdot 170$ & 0.080 \\
\hline Height $(\mathrm{cm})$ & -0.196 & 0.069 & -0.051 & 0.071 & -0.014 & 0.076 & -0.111 & 0.070 & -0.031 & 0.065 & -0.062 & 0.441 \\
\hline Lactation§ & -1.944 & $0.942^{*}$ & -0.587 & $0 \cdot 192^{* *}$ & -0.138 & 0.185 & -1.427 & 0.530 & -0.552 & $0.169^{* *}$ & -0.198 & 0.177 \\
\hline Age (years) & -0.096 & 0.130 & -0.082 & 0.135 & -0.021 & 0.141 & & & & & & \\
\hline Parity $(n) \|$ & +0.141 & 0.623 & +0.344 & 0.637 & +0.440 & 0.646 & & & & & & \\
\hline $25 \mathrm{OHD}(\mathrm{nmol} / \mathrm{l}) \dagger$ & -0.071 & $0.017^{\star \star \star}$ & -0.033 & 0.020 & -0.021 & 0.021 & -0.058 & $0.019^{\star *}$ & & & & \\
\hline Ca intake $(\mathrm{g} / \mathrm{d}) \boldsymbol{\pi} \dagger \dagger$ & -1.702 & 1.064 & -0.598 & $1 \cdot 137$ & +0.526 & $1 \cdot 197$ & & & & & & \\
\hline PAL†† & -5.638 & $2 \cdot 627^{\star}$ & $-2 \cdot 658$ & $2 \cdot 724$ & -0.288 & 3.08 & $-3 \cdot 331$ & $2 \cdot 406$ & & & & \\
\hline Oestrogen contraceptives††㧊 & +4.554 & $1.999^{\star}$ & +2.032 & 2.069 & -0.748 & 3.678 & +3.728 & 1.908 & & & & \\
\hline $\begin{array}{l}\text { Baseline lumbar spine } \\
\text { aBMD }\left(\mathrm{g} / \mathrm{cm}^{2}\right)\end{array}$ & -9.239 & $3.836^{*}$ & $-17 \cdot 201$ & $3.585^{\star \star \star}$ & $-14 \cdot 266$ & $3.786^{\star \star *}$ & -8.879 & $3.428^{\star}$ & $-17 \cdot 347$ & $\pm 3 \cdot 410^{\star * \star}$ & $-15 \cdot 128$ & $3 \cdot 815^{\star \star \star}$ \\
\hline
\end{tabular}

mo pp, months postpartum; 250HD, 25-hydroxyvitamin D; PAL, physical activity level.Units used are percentage change in the dependent variable per unit of the independent variable.

Percentage change in bone variables compared with baseline was calculated by the difference in log-transformed data between two time points, using log e and multiplied by 100 . Statistical analyses performed with univariable linear regression and adjusted multivariable linear regression.

Multivariable analyses always adjusted for lactation, body weight at baseline, change in body weight at time of measurement minus baseline and height.

${ }^{\star} P<0.05,{ }^{* \star} P<0.01,{ }^{\star \star \star} P<0.001$

$\dagger$ At baseline; at 2 weeks after delivery.

$\ddagger$ Compared with baseline (2 weeks after delivery).

$\S$ Lactation variables used, at 4 months postpartum if the women were fully lactating $(0=$ no, $1=$ yes), at 12 months postpartum duration of full lactation (months), at 18 months postpartum duration of total lactation (months).

II Number of births $(n)$.

I Total daily $\mathrm{Ca}$ intake, including both dietary and supplemental intake $(\mathrm{g} / \mathrm{d})$.

†† At 4 months postpartum (scale 1.3-2.2).

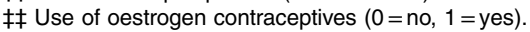

Table 3. Determinants for percentage change compared with baseline† in cortical volumetric bone mineral density (VBMD) at ultra-distal tibia postpartum ( $\beta$-Coefficients with their standard errors)

\begin{tabular}{|c|c|c|c|c|c|c|c|c|c|c|c|c|}
\hline \multirow[b]{3}{*}{ Determinants } & \multicolumn{6}{|c|}{ Univariable linear regression } & \multicolumn{6}{|c|}{ Multivariable linear regression } \\
\hline & \multicolumn{2}{|c|}{4 mo pp } & \multicolumn{2}{|c|}{$12 \mathrm{mo} \mathrm{pp}$} & \multicolumn{2}{|c|}{$18 \mathrm{mo} \mathrm{pp}$} & \multicolumn{2}{|c|}{4 mo pp } & \multicolumn{2}{|c|}{$12 \mathrm{mo} \mathrm{pp}$} & \multicolumn{2}{|c|}{$18 \mathrm{mo} \mathrm{pp}$} \\
\hline & $\beta$ & SEM & $\beta$ & SEM & $\beta$ & SEM & $\beta$ & SEM & $\beta$ & SEM & $\beta$ & SEM \\
\hline$n$ & \multicolumn{2}{|c|}{81} & \multicolumn{2}{|c|}{79} & \multicolumn{2}{|c|}{58} & \multicolumn{2}{|c|}{81} & \multicolumn{2}{|c|}{79} & \multicolumn{2}{|c|}{58} \\
\hline Body weight $(\mathrm{kg}) \dagger$ & +0.021 & $0.006^{* *}$ & +0.032 & $0.012^{* *}$ & +0.038 & $0 \cdot 13^{\star \star}$ & +0.022 & $0.008^{\star *}$ & +0.032 & $0.013^{*}$ & +0.032 & 0.017 \\
\hline Body weight change $(\mathrm{kg}) \ddagger$ & -0.031 & 0.017 & -0.007 & 0.027 & -0.027 & 0.029 & -0.003 & 0.019 & +0.023 & 0.027 & +0.007 & 0.300 \\
\hline Height $(\mathrm{cm})$ & +0.010 & 0.010 & +0.028 & 0.018 & +0.038 & 0.021 & -0.006 & 0.010 & 0.009 & 0.019 & 0.080 & 0.024 \\
\hline Lactation§ & -0.233 & 0.142 & -0.151 & $0.049^{* *}$ & -0.177 & $0.053^{*}$ & $-0 \cdot 160$ & 0.138 & -0.130 & $0.051^{*}$ & -0.079 & 0.510 \\
\hline Age (years) & -0.031 & 0.019 & -0.460 & 0.034 & +0.005 & 0.039 & & & & & & \\
\hline Parity $(n) \|$ & +0.082 & 0.090 & +0.099 & 0.161 & +0.064 & 0.181 & & & & & & \\
\hline $25 \mathrm{OHD}(\mathrm{nmol} / \mathrm{l}) \dagger$ & 0.000 & 0.003 & +0.004 & 0.005 & +0.030 & 0.006 & & & & & & \\
\hline Ca intake $(\mathrm{g} / \mathrm{d}) \mathrm{q} \dagger \dagger$ & +0.154 & 0.154 & +0.371 & 0.281 & +0.887 & $0.307^{\star *}$ & & & & & +0.688 & $0.060^{*}$ \\
\hline PAL†† & -0.323 & 0.410 & +0.313 & 0.724 & +0.364 & 0.824 & & & & & & \\
\hline Oestrogen contraceptives十†㧊 & +0.752 & $0.285^{*}$ & $+1 \cdot 124$ & $0.510^{*}$ & -0.467 & 1.024 & +0.628 & $0.294^{\star}$ & +0.621 & 0.534 & & \\
\hline Baseline cortical vBMD $\left(\mathrm{mg} / \mathrm{cm}^{2}\right)$ & +0.133 & 0.153 & +1.230 & $2 \cdot 737$ & $-4 \cdot 948$ & 3.259 & & & & & & \\
\hline
\end{tabular}

mo pp, months postpartum; 25OHD, 25-hydroxyvitamin D; PAL, physical activity level.Units used are percentage change in the dependent variable per unit of the independent variable.

Percentage change in bone variables compared with baseline was calculated by the difference in log-transformed data between two time points, using log $e$ and multiplied by 100 . Statistical analyses performed with univariable linear regression and adjusted multivariable linear regression.

Multivariable analyses always adjusted for lactation, body weight at baseline, change in body weight at time of measurement minus baseline and height.

${ }^{\star} P<0.05,{ }^{\star *} P<0.01$

$\dagger$ At baseline; at 2 weeks after delivery.

‡ Compared with baseline (2 weeks after delivery).

$\S$ Lactation variables used, at 4 months postpartum if the women were fully lactating $(0=$ no, $1=$ yes), at 12 months postpartum duration of full lactation (months), at 18 months postpartum duration of total lactation (months).

II Number of births.

I Total daily $\mathrm{Ca}$ intake, including both dietary and supplemental intake $(\mathrm{g} / \mathrm{d})$.

†† At 4 months postpartum (scale 1.3 to $2 \cdot 2$ ).

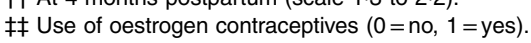


At 12 months postpartum, cortical vBMD at the ultra-distal tibia was still significantly lower than at baseline ( -0.62 (SD 0.11$) \%$, $P<0.001)^{(11)}$. Body weight at baseline $(P=0.015)$ was positively related to changes in cortical vBMD during the first 12 months postpartum, whereas an inverse relationship was found with duration of full lactation $(P=0 \cdot 013)$ (Table 3$)$, as analysed with multivariable linear regression model.

At 18 months postpartum, a significant decrease compared with baseline was still evident for cortical vBMD (-0.32 (SD 0.13)\%, $P<0.01)$ at the ultra-distal tibia ${ }^{(11)}$. Ca intake at 4 months $(P=0.029)$ was positively related to the changes in cortical vBMD during the first 18 months postpartum (Table 3), as analysed with multivariable linear regression model.

\section{Determinants of changes in cortical thickness at ultra-distal tibia}

For cortical thickness at the ultra-distal tibia, a significant decrease was observed during the first 4 months postpartum, compared with baseline $(-1.72$ (SD 0.84) \%, $P<0.05)$ (Fig. 1) $)^{(11)}$. No significant relationships were found between any of the investigated variables and changes in cortical thickness during the first 4 months postpartum (Table 4), as analysed with multivariable linear regression model.

At 12 months postpartum, a significant decrease compared with baseline was still evident for cortical thickness ( -0.87 (sD 0.33$) \%$, $P<0.01)^{(11)}$. Body weight at baseline $(P=0.001)$ was positively related, and duration of full lactation $(P=0.006)$ was inversely related, to the changes in cortical thickness (Table 4$)$, as analysed with multivariable linear regression model.
At 18 months postpartum, cortical thickness at the ultra-distal tibia was significantly higher compared with baseline $(+0.95$ (sD $0 \cdot 42) \%, P<0 \cdot 01)^{(11)}$. Positive relationships were evident for body weight at baseline $(P=0 \cdot 001)$ and $\mathrm{Ca}$ intake at 4 months postpartum $(P=0.017)$, and changes in cortical thickness during the first 18 months postpartum (Table 4 ), as analysed with multivariable linear regression model.

\section{Determinants of changes in trabecular thickness at ultra-distal tibia}

For trabecular thickness at the ultra-distal tibia, a non-significant decrease of -1.29 (SD 0.62)\% was observed during the first 4 months postpartum (Fig. 1) ${ }^{(11)}$. A significant inverse relation was found between baseline trabecular thickness and changes in trabecular thickness during the first 4 months postpartum $(P=0.022)$ (Table 5), as analysed with multivariable linear regression model.

At 12 months postpartum, trabecular thickness at the ultradistal tibia was significantly lower than baseline $(-2.06$ (sD 0.67$) \%, P<0.01)^{(11)}$. Significant inverse relationships were evident between baseline trabecular thickness $(P=0.011)$ and changes in trabecular thickness during the first 12 months postpartum (Table 5), as analysed with multivariable linear regression model.

At 18 months postpartum, trabecular thickness at the ultradistal tibia was still significantly lower compared with baseline $(-2.62$ (sD 0.94$) \%, P<0.01)^{(11)}$. At 18 months postpartum, only body weight at baseline $(P=0 \cdot 006)$ was positively related to the

Table 4. Determinants for percentage change compared with baseline† in cortical thickness at ultra-distal tibia postpartum ( $\beta$-Coefficients with their standard errors)

\begin{tabular}{|c|c|c|c|c|c|c|c|c|c|c|c|c|}
\hline \multirow[b]{3}{*}{ Determinants } & \multicolumn{6}{|c|}{ Univariable linear regression } & \multicolumn{6}{|c|}{ Multivariable linear regression } \\
\hline & \multicolumn{2}{|c|}{$4 \mathrm{mo} \mathrm{pp}$} & \multicolumn{2}{|c|}{$12 \mathrm{mo} \mathrm{pp}$} & \multicolumn{2}{|c|}{$18 \mathrm{mo} \mathrm{pp}$} & \multicolumn{2}{|c|}{$4 \mathrm{mo} \mathrm{pp}$} & \multicolumn{2}{|c|}{$12 \mathrm{mo} \mathrm{pp}$} & \multicolumn{2}{|c|}{$18 \mathrm{mo} \mathrm{pp}$} \\
\hline & $\beta$ & SEM & $\beta$ & SEM & $\beta$ & SEM & $\beta$ & SEM & $\beta$ & SEM & $\beta$ & SEM \\
\hline$n$ & \multicolumn{2}{|c|}{81} & \multicolumn{2}{|c|}{79} & \multicolumn{2}{|c|}{58} & \multicolumn{2}{|c|}{81} & \multicolumn{2}{|c|}{79} & \multicolumn{2}{|c|}{58} \\
\hline Body weight $(\mathrm{kg}) \dagger$ & +0.085 & 0.090 & +0.104 & $0.033^{\star *}$ & +0.123 & $0.041^{* *}$ & +0.102 & 0.110 & +0.131 & $0.036^{\star \star}$ & $+0 \cdot 175$ & $0.051^{* *}$ \\
\hline Body weight change $(\mathrm{kg}) \ddagger$ & -0.156 & 0.236 & +0.016 & 0.078 & -0.043 & 0.091 & -0.094 & 0.267 & +0.097 & 0.075 & $+0 \cdot 131$ & 0.090 \\
\hline Height $(\mathrm{cm})$ & -0.052 & 0.135 & +0.021 & 0.052 & +0.047 & 0.067 & -0.112 & 0.150 & -0.040 & 0.051 & $-0 \cdot 112$ & 0.071 \\
\hline Lactation§ & $-2 \cdot 132$ & 1.910 & -0.400 & $0 \cdot 141^{* *}$ & -0.409 & $0 \cdot 164^{*}$ & -2.087 & 1.934 & -0.381 & $0.133^{* *}$ & -0.293 & 0.152 \\
\hline Age (years) & +0.044 & 0.252 & -0.029 & 0.097 & -0.081 & 0.124 & & & & & & \\
\hline Parity $(n) \|$ & $-1 \cdot 768$ & $1 \cdot 182$ & +0.606 & 0.455 & +0.361 & 0.566 & & & & & & \\
\hline $250 \mathrm{OHD}(\mathrm{nmol} / \mathrm{l}) \dagger$ & +0.025 & 0.036 & -0.009 & 0.015 & +0.007 & 0.019 & & & & & & \\
\hline Ca intake $(\mathrm{g} / \mathrm{d}) \boldsymbol{\uparrow} \dagger \dagger$ & +0.861 & 2.072 & $+1 \cdot 141$ & 0.786 & $+2 \cdot 634$ & $0.968^{\star *}$ & & & & & $+2 \cdot 279$ & $0.920^{*}$ \\
\hline PAL†† & +2.427 & 5.477 & +1.403 & 2.036 & +1.359 & 2.593 & & & & & & \\
\hline Oestrogen contraceptives††ł‡ & $+2 \cdot 294$ & 3.928 & +1.442 & 1.490 & -1.616 & $3 \cdot 216$ & & & & & & \\
\hline Baseline cortical thickness (mm) & +3.165 & $4 \cdot 303$ & +0.394 & 1.652 & $-1 \cdot 135$ & $2 \cdot 061$ & & & & & & \\
\hline
\end{tabular}

mo pp, months postpartum; 25OHD, 25-hydroxyvitamin D; PAL, physical activity level.

Units used are percentage change in the dependent variable per unit of the independent variable.

Percentage change in bone variables compared with baseline was calculated by the difference in log-transformed data between two time points, using log $e$ and multiplied by 100 . Statistical analyses performed with univariable linear regression and adjusted multivariable linear regression.

Multivariable analyses always adjusted for lactation, body weight at baseline, change in body weight at time of measurement minus baseline and height.

${ }^{\star} P<0.05,{ }^{* \star} P<0.01$.

$\dagger$ At baseline; at 2 weeks after delivery.

$\ddagger$ Compared with baseline ( 2 weeks after delivery).

$\S$ Lactation variables used, at 4 months postpartum if the women were fully lactating $(0=$ no, $1=$ yes), at 12 months postpartum duration of full lactation (months), at 18 months postpartum duration of total lactation (months).

II Number of births.

II Total daily $\mathrm{Ca}$ intake, including both dietary and supplemental intake $(\mathrm{g} / \mathrm{d})$.

t† At 4 months postpartum (scale 1.3-2.2).

拉 Use of oestrogen contraceptives $(0=$ no, $1=$ yes $)$. 
Table 5. Determinants for percentage change compared with baseline† in trabecular thickness at ultra-distal tibia postpartum ( $\beta$-Coefficients with their standard errors)

\begin{tabular}{|c|c|c|c|c|c|c|c|c|c|c|c|c|}
\hline \multirow[b]{3}{*}{ Determinants } & \multicolumn{6}{|c|}{ Univariable linear regression } & \multicolumn{6}{|c|}{ Multivariable linear regression } \\
\hline & \multicolumn{2}{|c|}{4 mo pp } & \multicolumn{2}{|c|}{$12 \mathrm{mo} \mathrm{pp}$} & \multicolumn{2}{|c|}{$18 \mathrm{mo} \mathrm{pp}$} & \multicolumn{2}{|c|}{$4 \mathrm{mo} \mathrm{pp}$} & \multicolumn{2}{|c|}{$12 \mathrm{mo} \mathrm{pp}$} & \multicolumn{2}{|c|}{$18 \mathrm{mo} \mathrm{pp}$} \\
\hline & $\beta$ & SEM & $\beta$ & SEM & $\beta$ & SEM & $\beta$ & SEM & $\beta$ & SEM & $\beta$ & SEM \\
\hline$n$ & \multicolumn{2}{|c|}{81} & \multicolumn{2}{|c|}{79} & \multicolumn{2}{|c|}{58} & \multicolumn{2}{|c|}{81} & \multicolumn{2}{|c|}{79} & \multicolumn{2}{|c|}{58} \\
\hline Body weight $(\mathrm{kg}) \dagger$ & +0.115 & 0.065 & +0.123 & 0.070 & +0.281 & $0.092^{\star \star}$ & +0.085 & 0.080 & +0.100 & 0.082 & +0.353 & $0.122^{\star \star}$ \\
\hline Body weight change $(\mathrm{kg}) \ddagger$ & $-0 \cdot 108$ & 0.173 & -0.057 & $0 \cdot 161$ & -0.146 & 0.206 & -0.063 & 0.187 & +0.002 & 0.161 & $+0 \cdot 140$ & 0.180 \\
\hline Height $(\mathrm{cm})$ & -0.053 & 0.099 & -0.050 & 0.107 & +0.051 & 0.152 & -0.104 & 0.105 & -0.093 & 0.111 & -0.219 & 0.162 \\
\hline Lactation§ & -1.542 & 1.390 & -0.594 & $0.297^{\star}$ & -0.280 & 0.390 & -1.329 & 1.344 & -0.566 & 0.287 & -0.248 & 0.362 \\
\hline Age (years) & +0.006 & 0.186 & +0.245 & 0.198 & +0.348 & 0.277 & & & & & & \\
\hline Parity $(n) \|$ & +0.553 & 0.879 & +0.018 & 0.948 & +0.480 & 1.288 & & & & & & \\
\hline $25 \mathrm{OHD}(\mathrm{nmol} / \mathrm{l}) \dagger$ & -0.018 & 0.026 & -0.036 & 0.030 & +0.027 & 0.044 & & & & & & \\
\hline Ca intake $(\mathrm{g} / \mathrm{d}) \boldsymbol{q} \dagger \dagger$ & -1.454 & 1.499 & -0.560 & 1.681 & +0.293 & 2.395 & & & & & & \\
\hline PAL†† & -2.652 & $4 \cdot 000$ & +3.890 & $4 \cdot 270$ & +4.077 & 5.963 & & & & & & \\
\hline Oestrogen contraceptiv & -0.127 & 2.902 & -0.833 & 3.090 & -3.038 & $7 \cdot 298$ & & & & & & \\
\hline Baseline trabecular thickness $(\mathrm{mm})$ & -9.888 & $3 \cdot 777^{\star *}$ & -11.353 & $3.563^{\star \star}$ & -11.321 & $4.833^{*}$ & -8.414 & $3.598^{*}$ & -9.819 & $3.761^{*}$ & $-7 \cdot 362$ & 4.838 \\
\hline
\end{tabular}

mo pp, months postpartum; 25OHD, 25-hydroxyvitamin D; PAL, physical activity level.

Units used are percentage change in the dependent variable per unit of the independent variable.

Percentage change in bone variables compared with baseline was calculated by the difference in log-transformed data between two time points, using log $e$ and multiplied by 100 .

Statistical analyses performed with univariable linear regression and adjusted multivariable linear regression.

Multivariable analyses always adjusted for lactation, body weight at baseline, change in body weight at time of measurement minus baseline and height.

${ }^{\star} P<0.05,{ }^{* \star} P<0.01$.

$\dagger$ At baseline; at 2 weeks after delivery.

$\ddagger$ Compared with baseline ( 2 weeks after delivery).

$\S$ Lactation variables used, at 4 months postpartum if the women were fully lactating $(0=$ no, $1=$ yes), at 12 months postpartum duration of full lactation (months), at 18 months postpartum duration of total lactation (months).

\| Number of births.

I Total daily $\mathrm{Ca}$ intake, including both dietary and supplemental intake (g/d).

†† At 4 months postpartum (scale 1·3-2.2).

拉 Use of oestrogen contraceptives $(0=$ no, $1=$ yes $)$.

changes in trabecular thickness during the first 18 months postpartum (Table 5), as analysed using multivariable linear regression model.

\section{Discussion}

This is the first study investigating possible determinants, including $\mathrm{Ca}$ intake and serum $25 \mathrm{OHD}$, of the compartmental changes in bone microstructural and dimensional parameters and vBMD postpartum. Associations between serum 25OHD and changes in aBMD have, to our knowledge, not been studied before in postpartum women. We have previously shown that there are significant postpartum changes in aBMD in the lumbar spine and volumetric, dimensional and microstructural bone parameters in the ultra-distal tibia ${ }^{(11)}$. We believe that by investigating determinants of these changes in volumetric, microstructural and dimensional bone parameters new and important information is added to the field. In this group of women, we found that lactation and body weight were the main determinants for skeletal changes during the first 18 months postpartum. These were the variables in the investigated spectra of possible determinants that were robust in almost all analyses, independent of skeletal site. The novel findings were that a high total $\mathrm{Ca}$ intake at 4 months postpartum may protect against decreases in cortical vBMD and thickness, but not trabecular thickness, during the first 18 months postpartum. In addition, higher serum concentration of $25 \mathrm{OHD}$ was related to larger decreases in the trabecular-rich lumbar spine aBMD during the first 4 months postpartum.
During pregnancy, there are indications that maternal vitamin D status may influence the changes in bone mineral status ${ }^{(26)}$. Hypothetically, it is possible that also the recovery of bone minerals in the end of lactation or after weaning is less successful in women with low vitamin D status ${ }^{(27,28)}$. Thus, one may speculate that women with higher serum 25OHD have higher intestinal $\mathrm{Ca}$ uptake, and therefore are more able to supply the Ca needed for breast milk production. However, this was not supported by our study, as our results instead suggest that higher serum 25OHD was related to larger decreases in lumbar spine aBMD. Krebs et al. ${ }^{(13)}$ did not find a relationship between dietary vitamin $\mathrm{D}$ intake and $\mathrm{ABMD}$ at the lumbar spine or mid-radius postpartum at all. Similarly, Möller et al. ${ }^{(10)}$ observed that vitamin D intake was not related to the decreases in aBMD at the whole-body, lumbar spine or total hip sites during the first 4 months postpartum. Neither of these two studies investigated the relationship between the serum concentrations of $25 \mathrm{OHD}$ and the postpartum changes in aBMD. Vitamin D status is, during the summer months, mainly influenced by sunlight exposure ${ }^{(20,22)}$. We are, to our knowledge, the first to use serum 25OHD as a measurement of vitamin D status, when studying the association between vitamin $\mathrm{D}$ and postpartum bone changes. No evidence of significant interactions of serum 25OHD at baseline and lactation category at 4 months postpartum or season at baseline on the decreases in lumbar spine aBMD during the first 4 months postpartum was found.

Most previous observational studies have found that $\mathrm{Ca}$ intake during lactation has no relation to the decreases in aBMD measured at various sites postpartum ${ }^{(2,3,14)}$, except for Krebs 
et al. ${ }^{(13)}$ who reported that higher Ca intake was associated with higher lumbar spine aBMD postpartum. Also $\mathrm{Ca}$ intervention studies in lactating women $^{(15,29,30)}$ found either no effect of $\mathrm{Ca}$ supplementation on whole-body or lumbar spine aBMD during the first 6 months postpartum ${ }^{(29,30)}$ or only a transient positive effect of Ca supplementation, preventing the decreases in radial and lumbar spine aBMD postpartum ${ }^{(15)}$. No previous studies have investigated the role of $\mathrm{Ca}$ intake and changes in bone microstructure or in cortical bone specifically. We found that a higher total $\mathrm{Ca}$ intake at 4 months postpartum was significantly related to smaller decreases in cortical vBMD and larger increases in cortical thickness at 18 months postpartum compared with baseline at the ultra-distal tibia. Hence, total $\mathrm{Ca}$ intake was found to be a determinant for the changes in cortical vBMD and cortical thickness, but not trabecular thickness or the trabecular-rich lumbar spine aBMD, at 18 months postpartum compared with baseline.

At recruitment of these women during pregnancy, we see that $\mathrm{Ca}$ intake is high also during the third trimester $(1170 \mathrm{mg}$ $\mathrm{Ca} / \mathrm{d}$ in the third trimester and $42 \%$ of the women were taking Ca supplements; data not previously published). Hence, the observed effect is probably not explained by a higher $\mathrm{Ca}$ intake postpartum than during pregnancy. Interaction between $\mathrm{Ca}$ intake at 4 months postpartum and duration of total lactation was checked, but no significant interaction was found.

The general recommendation from the Swedish Medical Agency is not to use hormonal contraceptives containing oestrogen while lactating, as oestrogen decreases breast milk production $^{(31)}$. Therefore, it is not surprising that only a few women ( $5 \%$ at 4 months and $7 \%$ at 12 months postpartum) were using oestrogen contraceptives and that most of those were fully lactating for only a short period (not more than 1 month). Thus, the observed positive relation between use of oestrogen contraceptive and the changes in bone variables needs to be handled with caution. In our study, oestrogen contraceptive use could rather be a proxy for absence of lactation or lactation for a short period of time. During lactation and amenorrhoea, oestrogen levels are low, which has been suggested to contribute to the decreases in bone minerals postpartum $^{(13,29,32)}$. At the end of a period of long lactation or after weaning, oestrogen levels start to increase again, which also has been suggested to contribute to the recovery of the bone minerals ${ }^{(14,29)}$. Many previous studies have reported that longer duration of lactation is related to larger decreases in aBMD at several skeletal sites postpartum ${ }^{(2,3,5,7-10)}$. We found that women who were fully lactating at 4 months postpartum were somewhat older than women who were not fully lactating at 4 months postpartum, but not significantly $(33.4 \mathrm{v} .31 \cdot 8$ years). A possible influence of ageing on the observed bone changes therefore cannot be excluded.

Our finding of a relationship between a lower baseline body weight and larger decreases in cortical bone variables at the ultradistal tibia, and to a lesser extent also in trabecular thickness, confirms previous findings ${ }^{(19,33)}$. The suggested explanation is that a lower body weight results in a lower mechanical load on the skeleton, in combination with a lower body fat content, which in turn may reduce the peripheral oestrogen production ${ }^{(19,34)}$. Both these factors may contribute to a decrease in bone minerals.
The women in this study may not be representative of the general population of postpartum women at northern latitudes. In all, $80 \%$ of the women had 3 years or more of education at university level, compared with $37 \%$ in the general Swedish population of women in the same age group ${ }^{(35)}$. Mean body weight at 12 months postpartum was $65.3 \mathrm{~kg}$, which is lower than the weight in the same age group and sex in the general population of $67.0 \mathrm{~kg}^{(36)}$. Mean age at baseline was 32.9 years, which is slightly higher than comparable national data of pregnant women of 30.8 years $^{(37)}$. At baseline, none of the women smoked, whereas national data show that $4.6 \%$ of mothers of newborns smoke ${ }^{(38)}$. In all, $70 \%$ of the women were fully lactating at 4 months postpartum, compared with national data of $52 \%{ }^{(38)}$. This difference might partly be due to the more strict definition of full lactation by the Swedish National Board of Health and Welfare than that used in this study ${ }^{(38)}$. However, $88 \%$ of the women in this study were lactating to some extent at 4 months postpartum, compared with only $75 \%$ of the women in the whole country. As the women in this study weigh less, have higher education, lactate to a larger extent and have actively chosen to participate in the study, they may be more health conscious than the general population. Thus, the results need to be handled with care.

Weaknesses in the study were the sample size and the homogeneous sample population, which makes it difficult to generalise the results. Another weakness was that no control group consisting of age-matched non-pregnant and nonlactating women was included. Further, only a few women were using oestrogen contraceptives, and therefore we cannot make any firm conclusions about this exposure. Strengths of the study included the broad spectra of possible determinants studied, including serum 25OHD, the longitudinal data with four time points, the long follow-up of 18 months postpartum and the inclusion of results from both DXA and HR-pQCT. As far as we know, this is the first study investigating the determinants of compartmental changes in bone microstructural and dimensional parameters and vBMD in women postpartum.

In conclusion, lactation and body weight at baseline were the main determinants of skeletal changes during the first 18 months postpartum. Longer duration of lactation was associated with larger decreases in bone variables postpartum, whereas a higher baseline body weight was associated with smaller decreases in bone variables postpartum. Ca intake and serum concentrations of $25 \mathrm{OHD}$ appear to have different associations with cortical and trabecular bone.

\section{Acknowledgements}

The authors thank research nurse Ulrika Hjertonsson and Daniel Sundh for performing bone measurements, technical assistants Elisabeth Gramatkovski and Birgitha Arvidsson and research nurse Anna Folino for help with data collection and handling and Dr Ann Laskey for valuable comments.

This study was supported by The Swedish Research Council Formas (no. 2007-398 and 2009-1504) (H. A.); the graduate School Environment and Health (H. A.); the Swedish Nutrition Foundation (P. B.); Willhelm \& Martina Lundgrens Vetenskapsfond (P. B.); Magnus Bergvall foundation (H. A.); Fredrik and Ingrid Thuring 
foundation (H. A.); Olof Johannisson foundation (H. A.); The Swedish Society of Medicine (H. A.); Swedish Society for Medical Research (H. A.); Sahlgrenska University Hospital foundation (H. A.); Gustaf V and Queen Victoria's Freemason Foundation (H. A.); and Kvinnor \& Hälsa Foundation (H. A.). No funders had any role in the design, analysis or writing of this article.

H. A. designed the research, P. B. and H. A. conducted the research, C. O. and M. L. provided DXA and HR-pQCT methodology, P. B. performed statistical analyses, P. B. and H. A. wrote the paper and $\mathrm{P}$. B. had primary responsibility for the final content. All authors have read and approved the final manuscript.

The authors have no conflicts of interest.

\section{References}

1. Drinkwater BL \& Chesnut CH 3rd (1991) Bone density changes during pregnancy and lactation in active women: a longitudinal study. Bone Miner 14, 153-160.

2. Sowers M, Corton G, Shapiro B, et al. (1993) Changes in bone density with lactation. JAMA 269, 3130-3135.

3. Laskey MA, Prentice A, Hanratty LA, et al. (1998) Bone changes after 3 mo of lactation: influence of calcium intake, breast-milk output, and vitamin D-receptor genotype. Am J Clin Nutr 67, 685-692.

4. Holmberg-Marttila D, Sievanen H \& Tuimala R (1999) Changes in bone mineral density during pregnancy and postpartum: prospective data on five women. Osteoporos Int 10, 41-46.

5. Laskey MA \& Prentice A (1999) Bone mineral changes during and after lactation. Obstet Gynecol 94, 608-615.

6. Hopkinson JM, Butte NF, Ellis K, et al. (2000) Lactation delays postpartum bone mineral accretion and temporarily alters its regional distribution in women. $J$ Nutr 130, 777-783.

7. Karlsson C, Obrant KJ \& Karlsson M (2001) Pregnancy and lactation confer reversible bone loss in humans. Osteoporos Int 12, 828-834.

8. More C, Bettembuk P, Bhattoa HP, et al. (2001) The effects of pregnancy and lactation on bone mineral density. Osteoporos Int 12, 732-737.

9. Karlsson MK, Ahlborg HG \& Karlsson C (2005) Maternity and bone mineral density. Acta Orthop 76, 2-13.

10. Möller UK, Vieth Streym S, Mosekilde L, et al. (2012) Changes in bone mineral density and body composition during pregnancy and postpartum. A controlled cohort study. Osteoporos Int 23, 1213-1223.

11. Brembeck P, Lorentzon M, Ohlsson C, et al. (2015) Changes in cortical volumetric bone mineral density and thickness, and trabecular thickness in lactating women postpartum. $J$ Clin Endocrinol Metab 100, 535-543.

12. Melton LJ 3rd, Riggs BL, van Lenthe GH, et al. (2007) Contribution of in vivo structural measurements and load/ strength ratios to the determination of forearm fracture risk in postmenopausal women. J Bone Miner Res 22, 1442-1448.

13. Krebs NF, Reidinger CJ, Robertson AD, et al. (1997) Bone mineral density changes during lactation: maternal, dietary, and biochemical correlates. Am J Clin Nutr 65, 1738-1746.

14. Kolthoff N, Eiken P, Kristensen B, et al. (1998) Bone mineral changes during pregnancy and lactation: a longitudinal cohort study. Clin Sci (Lond) 94, 405-412.

15. Polatti F, Capuzzo E, Viazzo F, et al. (1999) Bone mineral changes during and after lactation. Obstet Gynecol 94, 52-56.

16. Affinito P, Tommaselli GA, di Carlo C, et al. (1996) Changes in bone mineral density and calcium metabolism in breastfeeding women: a one year follow-up study. I Clin Endocrinol Metab 81, 2314-2318.

17. Holick MF (2007) Vitamin D deficiency. N Engl J Med 357, 266-281.

18. Olausson H, Laskey MA, Goldberg GR, et al. (2008) Changes in bone mineral status and bone size during pregnancy and the influences of body weight and calcium intake. Am J Clin Nutr 88, 1032-1039.

19. Sowers M, Kshirsagar A, Crutchfield M, et al. (1991) Body composition, age and femoral bone mass of young adult women. Ann Epidemiol 1, 245-254.

20. Brembeck P, Winkvist A, Bååth M, et al. (2016) Determinants of changes in vitamin D status postpartum in Swedish women. Br J Nutr 115, 422-430.

21. World Health Organization (2001) Declaration of Helsinki ethical principles for medical research involving human subjects. Bull World Health Organ 79, 373-374.

22. Brembeck P, Winkvist A \& Olausson H (2013) Determinants of vitamin $D$ status in pregnant fair-skinned women in Sweden. Br J Nutr 110, 856-864.

23. National Food Agency (1997) Matmallen. Uppsala: National Food Agency.

24. DEQAS (2016) Vitamin D External Quality Assessment Scheme. www.deqas.org (accessed May 2016).

25. Cole TJ (2000) Sympercents: symmetric percentage differences on the $100 \log (\mathrm{e})$ scale simplify the presentation of $\log$ transformed data. Stat Med 19, 3109-3125.

26. Javaid MK, Crozier SR, Harvey NC, et al. (2005) Maternal and seasonal predictors of change in calcaneal quantitative ultrasound during pregnancy. J Clin Endocrinol Metab 90, 5182-5187.

27. Specker BL (1994) Do North American women need supplemental vitamin D during pregnancy or lactation? Am J Clin Nutr 59, 484S-490S; discussion 490S-491S.

28. Kovacs CS (2005) Calcium and bone metabolism during pregnancy and lactation. J Mammary Gland Biol Neoplasia 10, $105-118$

29. Kalkwarf HJ \& Specker BL (1995) Bone mineral loss during lactation and recovery after weaning. Obstet Gynecol 86, 26-32.

30. Prentice A (2000) Maternal calcium metabolism and bone mineral status. Am J Clin Nutr 71, 1312S-1316S.

31. The Medical Products Agency, Sweden (2005) Antikonception behandlingsrekommendation (Contraception - treatment guidelines). Information från Läkemedelsverket 7, 7-19.

32. McNeilly AS, Tay CC \& Glasier A (1994) Physiological mechanisms underlying lactational amenorrhea. Ann $N Y$ Acad Sci 709, 145-155.

33. Malpeli A, Apezteguia M, Mansur JL, et al. (2012) Calcium supplementation, bone mineral density and bone mineral content. Predictors of bone mass changes in adolescent mothers during the 6-month postpartum period. Arch Latinoam Nutr 62, 30-36.

34. Lindsay R, Cosman F, Herrington BS, et al. (1992) Bone mass and body composition in normal women. J Bone Miner Res $\mathbf{7}$, 55-63.

35. Statistics Sweden (2012) Educational attainment of the population 2010. www.scb.se (accessed May 2016).

36. Statistics Sweden (2013) BMI, vikt och längd - medelvärden 1988-89, 2008-2011 (BMI, body weight and height - averages 1988-89, 2008-2011). www.scb.se (accessed May 2016).

37. Statistics Sweden (2013) Äldre mammor vanligare förr (Older mothers were more common in the past). www.scb.se (accessed May 2016)

38. Statistics Sweden (2014) Breast-feeding and smoking habits among parents of infants born in 2012. Stockholm: The National Board of Health and Welfare. www.scb.se (accessed May 2016). 